\title{
Hypertrophy in Mesenchymal Stem Cell Chondrogenesis: Effect of TGF- $\beta$ Isoforms and Chondrogenic Conditioning
}

\author{
Michael B. Mueller ${ }^{a}$ b Maria Fischer ${ }^{a} \quad$ Johannes Zellner ${ }^{a} \quad$ Arne Berner $^{a}$ \\ Thomas Dienstknecht ${ }^{a} \quad$ Lukas Prantl $^{\mathrm{a}}$ Richard Kujat ${ }^{\mathrm{a}}$ Michael Nerlich ${ }^{\mathrm{a}}$ \\ Rocky S. Tuan ${ }^{b, c}$ Peter Angele ${ }^{a}$ \\ ${ }^{a}$ Department for Trauma Surgery, Regensburg University Medical Center, Regensburg, Germany; \\ ${ }^{b}$ Cartilage Biology and Orthopaedics Branch, National Institute of Arthritis and Musculoskeletal and \\ Skin Diseases, National Institutes of Health, Department of Health and Human Services, Bethesda, Md., \\ 'Department of Orthopaedic Surgery and Center for Cellular and Molecular Engineering, \\ University of Pittsburgh School of Medicine, Pittsburgh, Pa., USA
}

\section{Key Words}

Bone marrow stromal cell $\cdot$ Cartilage biology $\cdot$ Cartilage repair $\cdot$ Cell biology $\cdot$ Chondrogenic differentiation . Hypertrophy $\cdot$ Mesenchymal progenitor cell differentiation - Mesenchymal stem cells $\cdot$ Tissue engineering

\begin{abstract}
Induction of chondrogenesis in mesenchymal stem cells (MSCs) with TGF- $\beta$ leads to a hypertrophic phenotype. The hypertrophic maturation of the chondrocytes is dependent on the timed removal of TGF- $\beta$ and sensitive to hypertrophypromoting agents in vitro. In this study, we have investigated whether TGF- $\beta 3$, which has been shown to be more prochondrogenic compared to TGF- $\beta 1$, similarly enhances terminal differentiation in an in vitro hypertrophy model of chondrogenically differentiating MSCs. In addition, we tested the impact of the time of chondrogenic conditioning on the enhancement of hypertrophy. MSCs were chondrogenically differentiated in pellet culture in medium containing TGF- $\beta 1$ or TGF- $\beta 3$. After 2 or 4 weeks, chondrogenic medium was switched to hypertrophy-inducing medium for 2 weeks. Aggregates were analyzed histologically and biochemically on days 14,28 and 42 . The switch to hypertrophy medium
\end{abstract}

after 14 days induced hypertrophic cell morphology and significant increase in alkaline phosphatase activity compared to the chondrogenesis only control using both TGF- $\beta 1$ and TGF- $\beta 3$. After 28 days predifferentiation, differences between hypertrophic and control groups diminished compared to 14 days predifferentiation. In conclusion, chondrogenic conditioning with both TGF- $\beta$ isoforms similarly induced hypertrophy in our experiment and allowed the enhancement of the hypertrophic chondrocyte phenotype by hypertrophic medium. Enhancement of hypertrophy was seen more clearly after the shorter chondrogenic conditioning. Therefore, to utilize this experimental model as a tool to study hypertrophy in MSC chondrogenesis, a predifferentiation period of 14 days is recommended.

Copyright $\odot 2010$ S. Karger AG, Basel

\begin{tabular}{ll}
\hline Abbreviations used in this paper \\
\hline ALP & alkaline phosphatase \\
DMEM & Dulbecco's modified Eagle medium \\
DMMB & dimethylmethylene blue \\
DNA & deoxyribonucleic acid \\
GAG & sulfated glycosaminoglycan \\
MSC & mesenchymal stem cell \\
TGF & transforming growth factor
\end{tabular}

\section{KARGER}

(c) 2010 S. Karger AG, Basel

Fax +41613061234 E-Mail karger@karger.ch www.karger.com www.karger.com/cto
Dr. Michael B. Mueller

Department for Trauma Surgery

Regensburg University Medical Center

Franz-Josef-Strauss-Allee 11, DE-93042 Regensburg (Germany)

Tel. +49941944 6805, Fax +49941944 6806, E-Mail michaelbmueller@ web.de 


\section{Introduction}

Adult mesenchymal stem cells (MSCs) are a potential cell source for engineering musculoskeletal tissues including cartilage. Chondrogenic differentiation of MSCs has been shown in different matrix-based and matrix-free systems [Yoo et al., 1998; Johnstone and Yoo, 1999; Barry et al., 2001; Noth et al., 2002; Sekiya et al., 2002; Tuli et al., 2003; Song et al., 2004; Ichinose et al., 2005; Li et al., 2005; Lisignoli et al., 2005]. In the widely used pellet culture system, cells are packed at high density by centrifugation and chondrogenesis is induced by a strictly defined serumfree medium containing TGF- $\beta$ as chondro-inductive growth factor [Johnstone et al., 1998; Yoo et al., 1998]. During MSC chondrogenesis an early onset of expression of chondrocyte hypertrophy-associated genes, including type X collagen, alkaline phosphatase (ALP) and others, has been reported [Johnstone et al., 1998; Yoo et al., 1998; Sekiya et al., 2002; Mwale et al., 2006a, b; Pelttari et al., 2006]. In previous work, we have shown that an additional wide array of stage-specific genes, including growth factor receptors, extracellular matrix proteins, transcription factors and others, is regulated during MSC chondrogenesis in a manner very similar to what is found during embryonic skeletal development from mesenchymal condensation to endochondral ossification [Mueller and Tuan, 2008]. Moreover, exposure of chondrogenically differentiating MSCs to culture conditions that are known to influence hypertrophy in chondrocytes during endochondral ossification, for example in the growth plate, triggers the same response in chondrifying MSCs as in chondrocytes. Thyroid hormone induces hypertrophy while TGF- $\beta$ and dexamethasone are antihypertrophic [Mackay et al., 1998; Mueller and Tuan, 2008]. These observations strongly suggest that MSC chondrogenesis using the current culture conditions entails a similar developmental program as in embryonic limb development. During embryonic limb development, loose mesenchyme condenses and mesenchymal progenitor cells in the condensation center undergo chondrogenic differentiation. In contrast to phenotypically stable articular chondrocytes, the chondrogenic state in these cells is transient. During terminal differentiation, these cells become hypertrophic and undergo apoptosis. Blood vessels and osteoprogenitor cells invade the hypertrophic cartilage and give rise to bone [Goldring et al., 2006; Shimizu et al., 2007]. Vascular invasion and mineralization have also been reported for chondrogenic pellet cultures of MSCs after ectopic implantation in vivo [Pelttari et al., 2006]. This characteristic of MSC chondrogenesis is a major con- cern for the application of MSCs in cartilage tissue engineering for articular cartilage repair. Specifically, the goal in articular cartilage tissue engineering must be to generate stable hyaline cartilage and not transient cartilage like in endochondral ossification. Strategies to prevent or diminish the expression of hypertrophy in MSC chondrogenesis are clearly necessary.

The most commonly used medium in MSC chondrogenesis contains TGF- $\beta$ as the crucial chondrogenic factor. The three isoforms TGF- $\beta 1$, TGF- $\beta 2$ and TGF- $\beta 3$ are known to induce chondrogenesis and most investigators have used either TGF- $\beta 1$ or TGF- $\beta 3$ [Johnstone et al., 1998; Mackay et al., 1998; Yoo et al., 1998; Barry et al., 2001; Tuli et al., 2003; Mwale et al., 2006b; Pelttari et al., 2006; Mueller and Tuan, 2008]. Of these two commonly used isoforms, TGF- $\beta 3$ is reported to have a higher chondrogenic potential than TGF- $\beta 1$ and to lead to a more rapid differentiation [Barry et al., 2001]. It is, however, unclear if this faster and stronger differentiation is associated with a higher susceptibility for hypertrophy. Using an in vitro hypertrophy model [Mueller and Tuan, 2008], we have tested here whether the putatively stronger chondrogenic isoform, TGF- $\beta 3$, predisposes chondrogenic pellet cultures of MSCs to hypertrophy compared to TGF- $\beta 1$. In addition, the time of chondrogenic conditioning prior to induction of hypertrophy was varied. The reason for that is that also under standard chondrogenic conditions, differentiating MSCs express hypertrophy markers and it is unclear whether the differentiation state at which the aggregates are hit with the hypertrophic medium has impact on the expression of hypertrophy.

\section{Materials and Methods}

\section{Isolation of MSCs}

Bone marrow was aspirated from the iliac crest of 3 patients ( 2 male, 1 female; age 19-27) undergoing surgery that required autologous bone grafting with approval of the IRB of the University of Regensburg and written consent. Mesenchymal progenitor cells were isolated by Ficoll (Biochrom, Berlin, Germany) gradient centrifugation followed by plastic adhesion. Cells were grown in monolayer culture in Dulbecco's modified Eagle's medium (DMEM) low glucose (Invitrogen, Karlsruhe, Germany) with 10\% fetal calf serum (PAN Biotech $\mathrm{GmbH}$, Aidenbach, Germany) and antibiotics at $37^{\circ} \mathrm{C}$ with $5 \% \mathrm{CO}_{2}$ until $80 \%$ confluence, trypsinized and frozen for later use in liquid nitrogen. After thawing and monolayer expansion, cells were used for the experiments at passage 2 or 3 .

\section{Chondrogenic Differentiation}

Cells were trypsinized and seeded in V-bottomed 96-well polypropylene plates at 200,000 cells per well. Pellets were formed by 


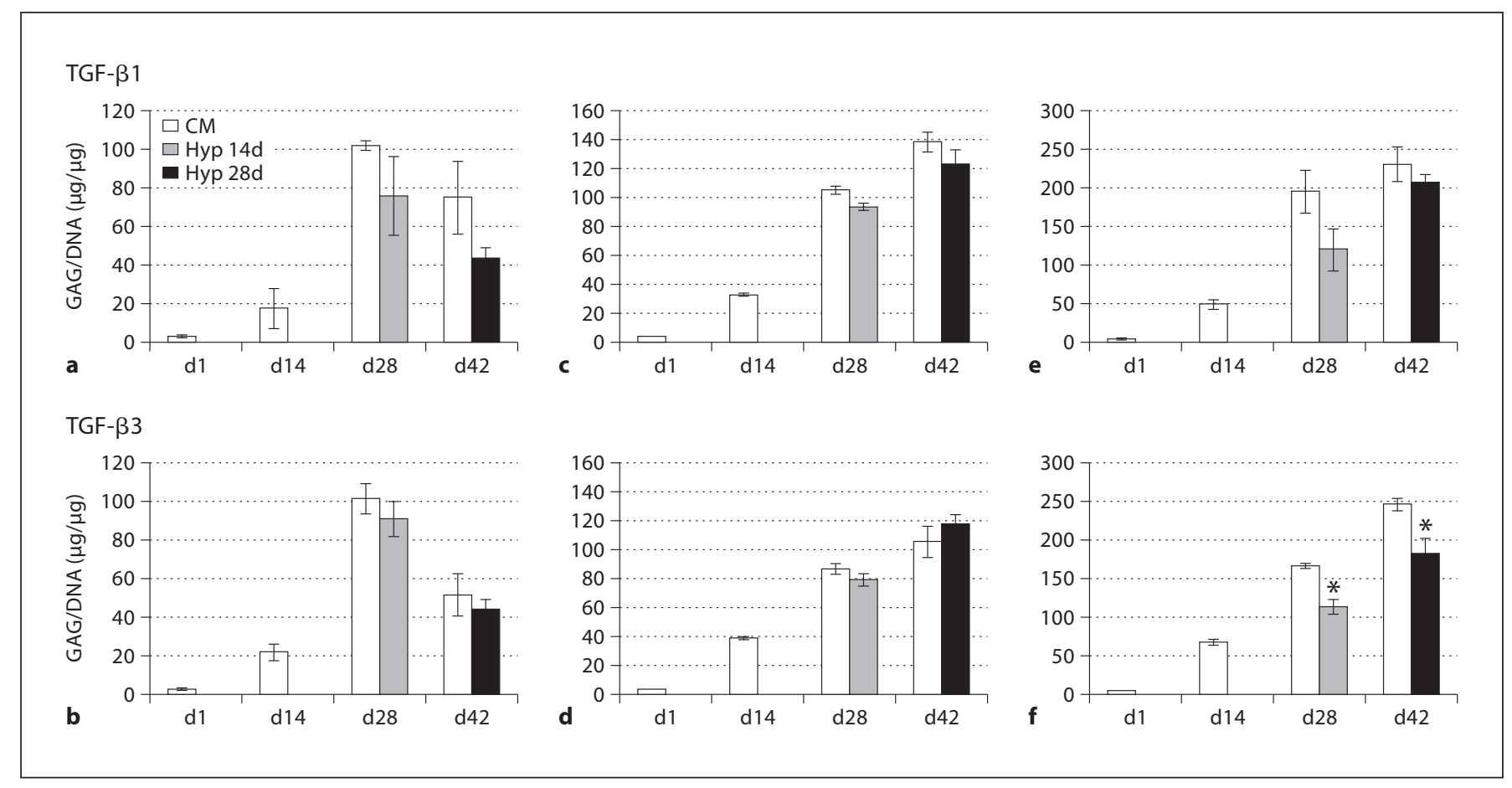

Fig. 1. Cartilage matrix production in chondrogenic pellet cultures of MSCs monitored as sulfated glycosaminoglycan content normalized to DNA content. Three independent experiments were performed with cells isolated from 3 different donors (a, b donor 1 ; c, d donor 2 ; e, f donor 3$)$ treated with TGF- $\beta 1$ (a, c, e) or TGF- $\beta 3(\mathbf{b}, \mathbf{d}, \mathbf{f})$. Open columns: chondrogenic control group (CM); grey columns: induction of hypertrophy after 14 days pre- differentiation (Hyp 14d); dark columns: induction of hypertrophy after 28 days pre-differentiation (Hyp 28d). Error bars show standard deviation; * significantly different from chondrogenic control $(\mathrm{p}<0.05) ; \mathrm{n}=4$. Reproducible significant differences between the two TGF- $\beta$ isoforms were not detected. Significant differences between hypertrophic and control groups were only detected in one cell population with TGF- $\beta 3$ (f). centrifugation at $250 \mathrm{~g}$ for $5 \mathrm{~min}$ and chondrogenically differentiated in DMEM with high glucose content (Invitrogen), 1\% ITS+3, $50 \mu \mathrm{g} / \mathrm{ml}$ ascorbate-2-phosphate, $40 \mu \mathrm{g} / \mathrm{mll}$-proline, $100 \mathrm{nM}$ dexamethasone (Sigma Aldrich, Steinheim, Germany) and $10 \mathrm{ng} / \mathrm{ml}$ TGF- $\beta 1$ or TGF- $\beta 3$ (R\&D Systems, Wiesbaden, Germany). After a predifferentiation period of either 14 or 28 days, medium conditions were changed to hypertrophy-enhancing medium [DMEM high glucose, $1 \% \mathrm{ITS}+3,50 \mu \mathrm{g} / \mathrm{ml}$ ascorbate-2-phosphate, $40 \mu \mathrm{g} /$ ml l-proline, $1 \mathrm{~nm}$ triiodothyronine (T3; Sigma Aldrich)], the control was kept in chondrogenic medium for the whole culture period. The medium was changed 3 times per week. Aggregates were harvested on days 1, 14, 28 and 42 for histological and biochemical analysis. In order to assess matrix mineralization in the chondrogenic and hypertrophic cultures, $5 \mathrm{~mm} \beta$-glycerophosphate (Sigma Aldrich) was added to the medium upon induction of hypertrophy in some of the hypertrophic and control aggregates.

\section{Histology, Histochemistry and Immunohistochemistry}

Aggregates were harvested on days 1, 14, 28 and 42, and either fixed in $4 \%$ paraformaldehyde and embedded in paraffin or frozen sections were prepared. Sections were stained with dimethylmethylene blue (DMMB) and von Kossa (both from Sigma Aldrich). Histochemical ALP staining with neutral red counterstain was carried out on frozen sections with a kit (Sigma Aldrich). For immunohistochemical detection we used commercially available antibodies for type I collagen (Sigma Aldrich), type II collagen (Merck, Darmstadt, Germany) and type X collagen (Quartett Immunodiagnostika und Biotechnologie $\mathrm{GmbH}$, Berlin, Germany). In brief, after antigen retrieval with pepsin digestion $(0.1 \%, \mathrm{pH} 3$, room temperature, $15 \mathrm{~min}$ ) for types I and II collagen and additional hyaluronidase digestion $\left(0.2 \%, \mathrm{pH}, 37^{\circ} \mathrm{C}, 60 \mathrm{~min}\right)$ for type $\mathrm{X}$ collagen and blocking (10\% fetal bovine serum/10\% goat se$\mathrm{rum} / \mathrm{PBS}$ ), incubation with the primary antibody was carried out at $4{ }^{\circ} \mathrm{C}$ overnight. Immunolabeling was detected with a biotinylated secondary antibody (Dianova, Hamburg, Germany), horse reddish peroxidase conjugated streptavidin (Vector Laboratories, Burlingame, Calif., USA) and metal enhanced diaminobenzidine as substrate (Sigma Aldrich).

\section{Biochemical Analysis}

Sulfated glycosaminoglycan (GAG) content normalized to DNA was used as a quantitative differentiation marker and ALP activity in the culture medium was used as a quantitative marker for hypertrophy. For determination of GAG and DNA content, aggregates were harvested on days 1, 14, 28 and 42 and digested in Sigma papain digestion solution $(150 \mu \mathrm{g} / \mathrm{ml}$ in PBS, $6 \mathrm{~mm}$ cysteine $\mathrm{HCl}, 6 \mathrm{mM}$ EDTA, pH 6.0) at $60^{\circ} \mathrm{C}$ overnight. Digestion was carried out in 4 replicates per time point and condition with 2 ag- 


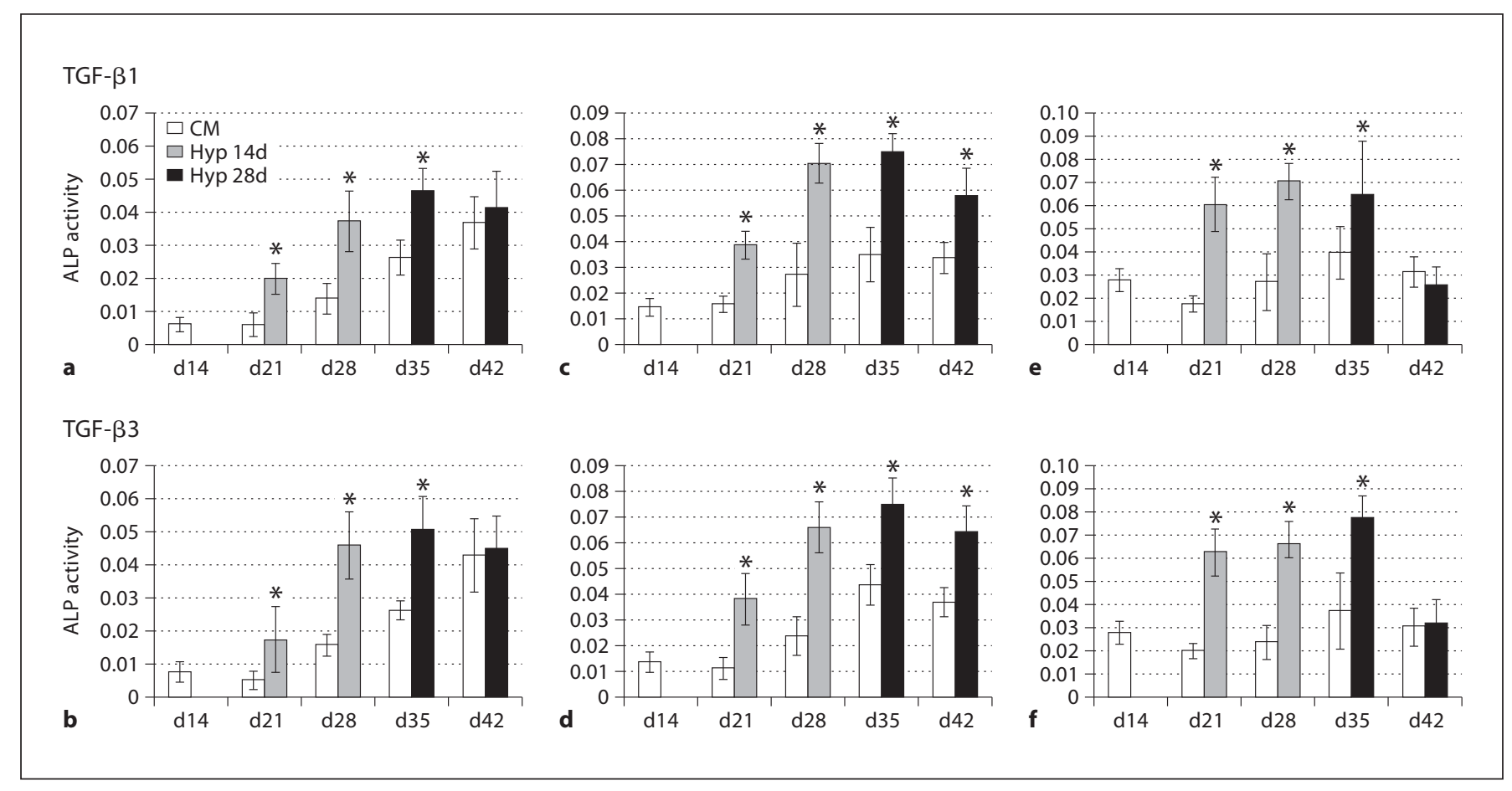

Fig. 2. Hypertrophy in chondrogenic pellet cultures monitored on the basis of ALP activity in the culture medium. Three independent experiments were performed with cells isolated from 3 different donors (a, b donor $1 ; \mathbf{c}, \mathbf{d}$ donor $2 ; \mathbf{e}, \mathbf{f}$ donor 3 ) treated with TGF- $\beta 1(\mathbf{a}, \mathbf{c}, \mathbf{e})$ or TGF- $\beta 3$ (b, d, f). Open columns: chondrogenic control group $(\mathrm{CM})$; grey columns: induction of hypertrophy after 14 days pre-differentiation (Hyp 14d); dark columns: induction of hypertrophy after 28 days pre-differentiation (Hyp 28d).

gregates in each digest. DNA content was determined with a Pico Green kit (Invitrogen) and GAG content with the dimethylmethylene blue method and chondroitin sulfate A from bovine trachea (Sigma Aldrich) as standard. For determination of ALP activity in the medium, medium was harvested on days 14, 21, 28, 35 and 42 , spun for $5 \mathrm{~min}$ at maximum speed, and $75 \mu \mathrm{l}$ of supernatant was incubated with ALP-buffer (1.5 M Tris, $1 \mathrm{mM} \mathrm{ZnCl}_{2}, 1 \mathrm{mM}$ $\mathrm{MgCl}_{2}, \mathrm{pH}$ 9.0) as well as p-nitrophenol phosphate (Sigma Aldrich) as substrate at a final concentration of $2 \mathrm{mg} / \mathrm{ml}$ at room temperature using microtiter plates in a plate reader (Genius plate reader; Tecan, Crailsheim, Germany). Continuous absorbance measurements at $405 \mathrm{~nm}$ were carried out and change in $\mathrm{A}_{405}$ over time $(\mathrm{dA} / \mathrm{min})$ calculated in the linear range of the reaction. For determination of the ALP activity, 8 replicates per time point and condition were used. For the GAG and DNA assays, 4 replicates per time point and condition were used.

\section{Statistical Analysis}

Statistical analysis was carried out by pairwise comparisons using unpaired, 2-tailed t test in Microsoft Excel.
Error bars show standard deviation; * significantly different from chondrogenic control $(\mathrm{p}<0.05) ; \mathrm{n}=8$. Significantly higher ALP activity was measured in the culture medium in the hypertrophic groups on day 21, day 28 and day 35 in all cell populations and on day 42 in one cell population (donor 2 ; c, d). No reproducible significant differences were detected between the two TGF- $\beta$ isoforms.

\section{Results}

DNA content did not change significantly over time. Pairwise comparisons of the DNA content between the control and respective hypertrophic groups as well as between the TGF- $\beta 1$ and the respective TGF- $\beta 3$ groups did not show any significant differences at all time points. Also, DNA content did not change significantly over time. The results of the GAG assay normalized to DNA are shown in figure 1. Significant difference in GAG/ DNA content between hypertrophic and control group was only seen in the TGF- $\beta 3$ group in cells from one donor (fig. 1f). Statistical comparison of the respective conditions with TGF- $\beta 1$ and TGF- $\beta 3$ as chondrogenic growth factor did not show any reproducible significant difference. The only significant difference was a higher GAG content normalized to DNA in the chondrogenic group on day 42 in one cell population ( $p=0.012$, cell donor 2; fig. 1c, d). The difference in nonnormalized 

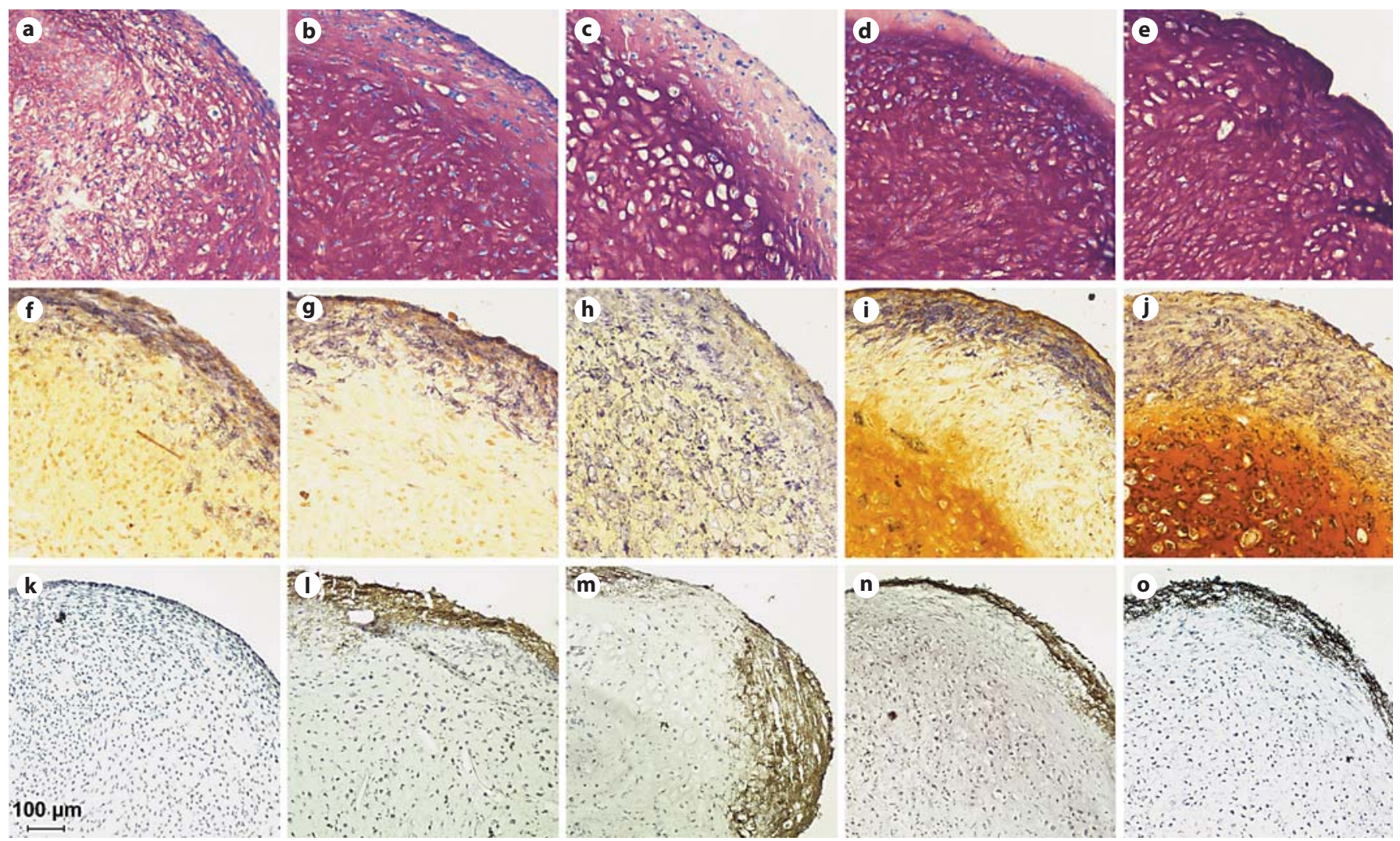

Fig. 3. Histological and histochemical analysis of chondrogenic pellet cultures of MSCs treated with TGF- $\beta 1$. DMMB (a-e), ALP $(\mathbf{f}-\mathbf{j})$ and von Kossa (k-o) staining of control chondrogenic cultures on day $14(\mathbf{a}, \mathbf{f}, \mathbf{k})$, day $28(\mathbf{b}, \mathbf{g}, \mathbf{I})$ and day $42(\mathbf{d}, \mathbf{i}, \mathbf{n})$ and of day 28 cultures with hypertrophic treatment started on day 14 (c, h, $\mathbf{m}$ ) and day 42 cultures with hypertrophic treatment started on day $28(\mathbf{e}, \mathbf{j}, \mathbf{o})$. Selected day 28 and day 42 cultures for von Kossa staining $(\mathbf{I}, \mathbf{m})$ also received $\beta$-glycerophosphate supplementation starting on day 14 for day 28 cultures and on day 28 for day 42 cultures. DMMB staining shows the typical hypertrophic morphology with large lacunae in the hypertrophic group on day 28 (c). ALP activity is located in the periphery of cultures in standard chondrogenic medium $(\mathbf{f}, \mathbf{g}, \mathbf{i})$ and positive throughout the entire aggregates in hypertrophic medium $(\mathbf{h}, \mathbf{j})$. Mineralization upon addition of $\beta$-glycerophosphate is restricted to the periphery of both control and hypertrophic cultures (I-o).
GAG content between TGF- $\beta 1$ and TGF- $\beta 3$ was not significant for any of the cell populations and conditions.

ALP activity in the culture medium was significantly higher in the hypertrophic groups compared to the respective control on days 21, 28 and 35 (fig. 2). On day 42, ALP activity in the medium was significantly higher in the hypertrophic group compared to the control group in only 1 of the 3 donors (donor 2; fig. $2 \mathrm{c}$, d). In the chondrogenic groups, ALP secretion increased until day 35 and then plateaued or increased further. In the hypertrophic groups, ALP secretion plateaued or decreased after day 35. Direct comparison of TGF- $\beta 1$ and TGF- $\beta 3$ in the respective groups did not show any reproducible trend or reproducible significant difference. ALP activity in the medium was significantly higher in the TGF- $\beta 3$ group in the control on day 28 in cell population $1(\mathrm{p}=0.037) \mathrm{com}$ - pared to TGF- $\beta 1$ control group on day 28 (fig. $2 \mathrm{a}, \mathrm{b}$ ). In the hypertrophic group with TGF- $\beta 3$ on day 35 compared to the hypertrophic group with TGF- $\beta 1$ on day 35 , ALP activity in the medium was significantly higher in cell population 2 ( $p=0.041$; fig. $2 c, d)$. These two statistically significant differences amounted to approximately $20 \%$.

Histologically, no differences between cultures treated with the two growth factor isoforms, TGF- $\beta 1$ and TGF$\beta 3$, could be detected. The histologies shown are from cultures that were treated with TGF- $\beta 1$. DMMB staining (fig. 3a-e) showed metachromatic staining of the extracellular matrix at all time points indicating chondrogenic differentiation and production of cartilage matrix. On day 28, after 14 days of chondrogenic predifferentiation, the cell morphology in the hypertrophic group (fig. 3c) 

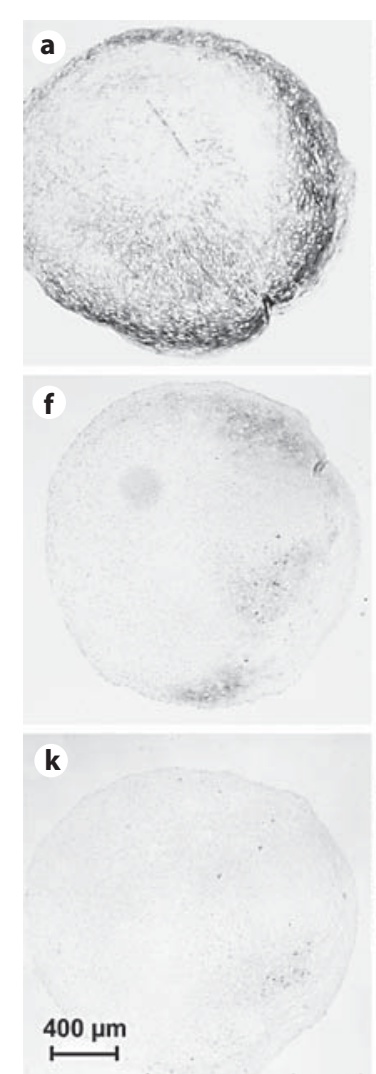

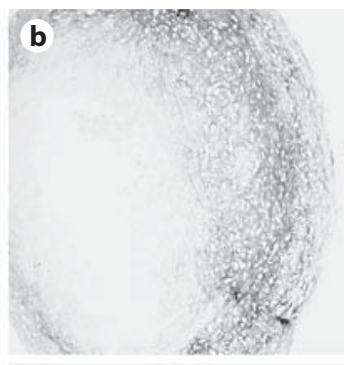

g
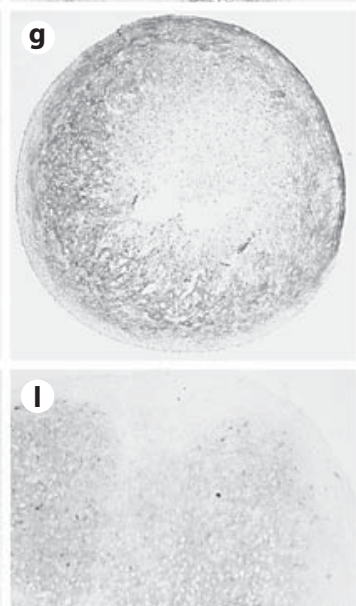
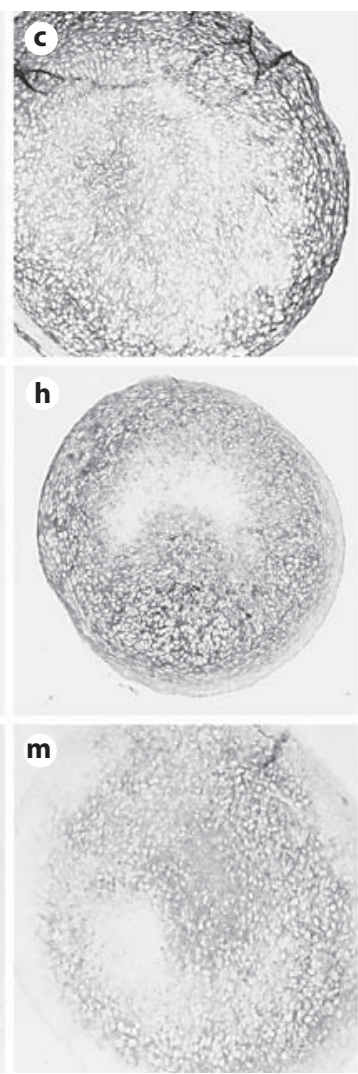
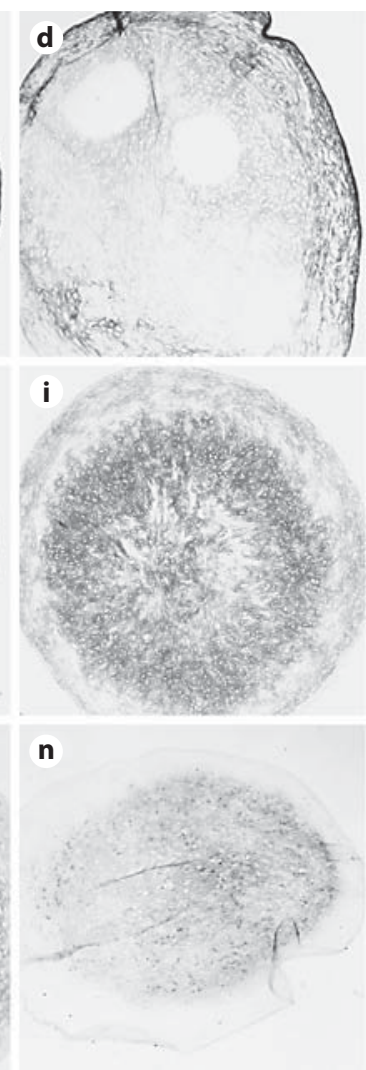
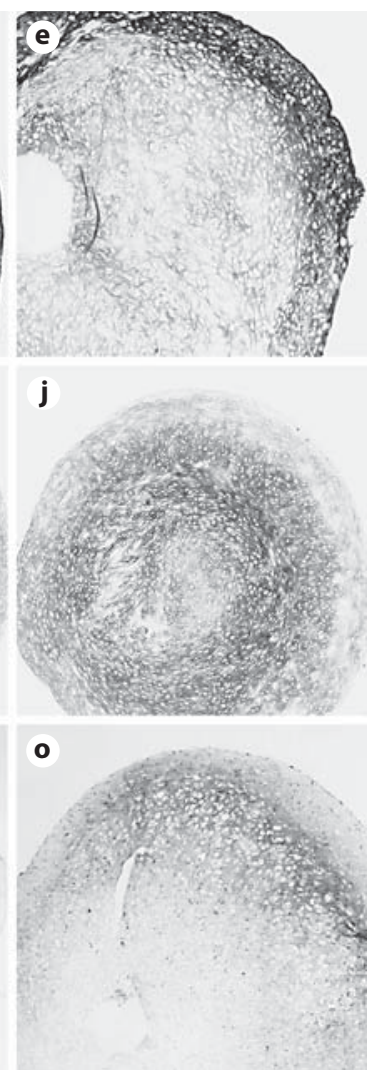

Fig. 4. Immunohistochemical analysis of matrix composition of chondrogenic pellet cultures of MSCs treated with TGF- $\beta 1$. Staining for type I (a-e), type II (f-j) and type X collagen (k-o) of control chondrogenic cultures on day $14(\mathbf{a}, \mathbf{f}, \mathbf{k})$, day $28(\mathbf{b}, \mathbf{g}, \mathbf{I})$ and day $42(\mathbf{d}, \mathbf{i}, \mathbf{n})$ and of hypertrophic cultures on day 28 after 14 days of chondrogenic pre-differentiation $(\mathbf{c}, \mathbf{h}, \mathbf{m})$ and day 42 after 28 days of chondrogenic pre-differentiation $(\mathbf{e}, \mathbf{j}, \mathbf{o})$. Strong type I collagen staining in the periphery of the cultures (a-e). Type I col- lagen staining in the center of the aggregates is stronger in hypertrophic (c, e) than control cultures (a, b, d). Initial type II collagen expression is detected on day 14 (f) and strong staining is observed on day 28 and day 42 in both control and hypertrophic cultures ( $\mathbf{g}-\mathbf{j})$. Type X collagen staining is negative on day $14(\mathbf{k})$ and positive in the hypertrophic $(\mathbf{m}, \mathbf{o})$ and in the control cultures $(\mathbf{I}, \mathbf{n})$ on day 28 and day 42 . was clearly different from that in the chondrogenic control (fig. 3b), with larger lacunae typical for hypertrophic cartilage. On day 42, after 28 days of chondrogenic predifferentiation, the morphological difference between the two groups was less clear.

ALP histochemical staining (fig. $3 \mathrm{f}-\mathrm{j}$ ) showed that ALP-positive cells were detected in the periphery of the chondrogenic aggregates on days 14, 28 and 42 (fig. 3f, g, i) and throughout the aggregates in the hypertrophic groups on both day 28 and day 42 (fig. $3 \mathrm{~h}, \mathrm{j}$ ).

Von Kossa staining was carried out on hypertrophic and control samples that received medium containing $\beta$ glycerophosphate as a substrate for mineralization from day $14 \mathrm{on}$. The samples examined included day $14 \mathrm{cul}-$ tures (fig. $3 \mathrm{k}$ ), which have not received any $\beta$-glycerophosphate and serve as a negative control. Both control (fig. 3l) and hypertrophic (fig. 3m) day 28 cultures had received $\beta$-glycerophosphate from day 14 on, and control (fig. 3n) and hypertrophic (fig. 3o) day 42 cultures had received $\beta$-glycerophosphate from day 28 on. The day 28 and 42 nonhypertrophic control group (fig. 3l, n) both showed mineralization of the peripheral ring where the ALP-positive cells were located (fig. 3g, i). In absence of $\beta$-glycerophosphate, day 14 aggregates did not mineralize at all. Interestingly, in the day 28 and day 42 hypertrophic groups (fig. $3 \mathrm{~m}, \mathrm{o}$ ), mineralization was also mainly restricted to the periphery. On some sections, the mineralized area extended slightly further to the center of the aggregates compared to the control, but this was not detected in all sections.

Immunohistochemical staining showed high type I collagen expression in the periphery of the aggregates in 
all groups at all time points (fig. $4 \mathrm{a}-\mathrm{e}$ ). Both on day 28 and day 42 , the staining in the central areas of the aggregates was stronger in the hypertrophic (fig. 4c, e) than the control group (fig. 4b, d). Type II collagen staining was still weak on day 14 (fig. 4f) and clearly positive on days 28 and 42 in both chondrogenic and hypertrophic groups, and was localized to the central area (fig. $4 \mathrm{~g}-\mathrm{j}$ ). Immunohistochemical detection of type $\mathrm{X}$ collagen was negative on day 14 (fig. $4 \mathrm{k}$ ) and positive on days 28 and 42 in both control and hypertrophic groups (fig. 4l-o). Staining intensity between control and hypertrophic groups differed only slightly between control and hypertrophic groups. Type X collagen was localized in the center of the aggregates with type II collagen and was negative in the periphery.

\section{Discussion}

We could reproducibly enhance the hypertrophic phenotype of chondrifying MSCs by switching from the standard chondrogenic to the hypertrophic condition in this experiment after predifferentiation with both TGF$\beta 1$ and TGF- $\beta 3$. This could clearly be shown by the different cell morphology after hypertrophy induction as well as the expression of hypertrophy markers such as ALP and type X collagen. Histologically and by quantification of ALP secretion, we did not detect differences between aggregates that have been preconditioned with TGF- $\beta 1$ or TGF- $\beta 3$. Our results are based on phenotypical analyses such as histology, immunohistochemistry and biochemical assays. Using these techniques facilitates the detection of relevant phenotypical differences between the groups. Although molecular analyses may yield additional results, their relevance to the actual phenotype is unknown, given our histological, immunohistochemical and biochemical results. Nevertheless, more sensitive assays like gene expression analysis could possibly detect differences between the two growth factor isoforms.

Hypertrophy markers are also expressed under standard chondrogenic culture conditions. Type X collagen staining is positive in the chondrogenic control groups on day 28 and 42 as well. Strikingly, ALP-positive cells, even at the latest time point (42 days), are restricted to the periphery of the aggregates in the chondrogenic control groups, whereas they are evenly distributed throughout the aggregates in the hypertrophic groups. Both type $\mathrm{X}$ collagen and ALP are markers of hypertrophic chondrocytes in the growth plate. There is some indication in the literature that the onset of type X collagen expression shortly precedes the onset of ALP expression in growth plate chondrocytes [Poole et al., 1989; Cheung et al., 2001], while other reports suggest a simultaneous regulation of these genes [Farquharson and Whitehead, 1995; Iwamoto et al., 1995]. For costal cartilage there is clear evidence that type X collagen and ALP are regulated differentially with an early onset of type X collagen expression during development, whereas the expression of ALP and endochondral ossification do not initiate before puberty [Bahrami et al., 2001]. Given this reported finding and the differential expression of type $\mathrm{X}$ collagen and ALP observed here, our results may be interpreted in the following manner. Under standard chondrogenic conditions, a developmental program similar to endochondral ossification in limb development is initiated by the strictly defined chondrogenic medium containing TGF- $\beta$ and dexamethasone. Previously published data support this hypothesis [Mueller and Tuan, 2008]. In standard chondrogenic medium, chondrifying MSCs mature to a type $\mathrm{X}$ collagen-positive but ALP-negative hypertrophic stage. However, under standard chondrogenic conditions, MSCs that have differentiated in vitro in aggregate culture display characteristics of hypertrophic cartilage, such as vascular invasion and ossification, when ectopically implanted in vivo [Pelttari et al., 2006]. Both TGF- $\beta$ and dexamethasone inhibit hypertrophy in chondrocytes [Ballock et al., 1993; Leboy et al., 1997], and thyroid hormone enhances hypertrophy [Quarto et al., 1997; Okubo and Reddi, 2003; Mello and Tuan, 2006] during endochondral ossification. In the pellet culture system used here, the withdrawal of the antihypertrophic agents, TGF- $\beta$ and dexamethasone, is a prerequisite for the enhancement of hypertrophy by the addition of thyroid hormone [Mueller and Tuan, 2008]. We therefore believe that the continuous application of TGF- $\beta$ and dexamethasone in the standard chondrogenic medium either slows down maturation or does not allow the transition from a type X collagen-positive and ALP-negative hypertrophic phenotype to a hypertrophic phenotype that expresses both type X collagen and ALP. When the culture medium is switched to the hypertrophic medium, the maturation restraining factors TGF- $\beta$ and dexamethasone are removed from the system and prohypertrophic thyroid hormone is added. This combination leads to either a massive acceleration of the maturation process or allows maturation to progress.

The difference between the hypertrophic group and the control group was clearly less distinct after 28 days of chondrogenic predifferentiation compared to the shorter 
predifferentiation period of 14 days. This is true both for the quantitative hypertrophy marker (ALP secretion) and the cell morphology. After 14 days, the switch to the hypertrophic medium induced a clearly hypertrophic cell phenotype represented by large lacunae, whereas after 28 days predifferentiation, there was almost no difference in cell morphology between the hypertrophic and the control group. Immunohistochemical type $\mathrm{X}$ collagen staining and histochemical alkaline phosaphatase activity, however, turned clearly positive after hypertrophy induction both after 14 and 28 days chondrogenic preconditioning. The increase in cell volume in hypertrophic chondrocytes of the growth plate is a very dynamic process and is mainly caused by an increase in organelles and an expression of increased metabolic activity [Hunziker et al., 1999; Ballock and O'Keefe, 2003]. We believe that this is also true for the enhancement of hypertrophy in MSC chondrogenesis. A possible reason for the lack of increase in cell volume after 28 days of predifferentiation is that cells may have reached a mature state in which they simply cannot react to the new prohypertrophic culture condition with a similar increase in metabolic activity as earlier in the chondrogenic differentiation, that is, on day 14 .

The presence of ALP activity in the periphery of the aggregates in the control group is unlikely to be an expression of hypertrophy. ALP activity is co-localized with type I collagen and not with type II and type X collagen, and is associated with the peripheral fibroblast-like cells that resemble periosteal or bone cells. This explanation is supported by the fact that these cells are associated with a mineralized matrix in the periphery of the aggregates upon supply of $\beta$-glycerophosphate. In the hypertrophic groups, we had expected mineralization of the entire aggregate after addition of $\beta$-glycerophosphate because of the strong homogeneous ALP activity detected throughout the aggregates. However, hypertrophic aggregates did not mineralize entirely after addition of $\beta$-glycerophosphate. Only on some sections the mineralization was extended further towards the center of the aggregates, compared to the controls. In previous work, we were able to detect mineralization of large amounts of the extracellular matrix after induction of hypertrophy and addition of $\beta$-glycerophospate [Mueller and Tuan, 2008]. The major differences between these studies are, first, the use of a different cell source with younger cell donors in the present study and, second, in the previous experiment, the hypertrophic medium contained $1 \mathrm{nM}$ dexamethasone but was withdrawn completely in the present study. Furthermore, in the previous study, the hypertrophic group was characterized by a certain histological heterogeneity with regions of undifferentiated and hypertrophic areas, which possibly allowed better tissue penetration of $\beta$ glycerophosphate because of the less dense extracellular matrix in undifferentiated areas.

In summary, we could not detect any difference between TGF- $\beta 1$ and TGF- $\beta 3$ in terms of susceptibility to the development of hypertrophy. Also, under standard chondrogenic conditions, differentiating MSCs developed a hypertrophic phenotype that expresses type X collagen but not ALP. This hypertrophic phenotype could reproducibly and significantly be enhanced after 2 weeks of chondrogenic predifferentiation with the hypertrophic conditions which could be shown by the different cell morphology and ALP positivity in the central, chondrogenic differentiated areas of the aggregates. After a longer predifferentiation period of 4 weeks, the difference between hypertrophic and chondrogenic aggregates was less distinct, in particular, the changed cell morphology with increase in cell volume was less obvious.

For MSC-based tissue engineering of articular cartilage, the expression of hypertrophy markers is a major concern because hypertrophy will ultimately lead to apoptosis, vascular invasion, and ossification of the tissue. The model described here is a useful tool for investigating mechanisms leading to hypertrophy and to test for culture conditions that may inhibit hypertrophy. Our data indicate that for these purposes, the choice of the TGF- $\beta$ isoform is not a critical issue, but that a predifferentiation period of 2 weeks is preferable over a predifferentiation period of 4 weeks because of the larger differences observed between the hypertrophic and control groups.

\section{Acknowledgements}

This study was supported by AO Research Fund (S-07-3M), German Research Foundation (DFG; MU2318-1) and the NIH Intramural Research Program (Z01 AR41131).

References

Bahrami, S., U. Plate, R. Dreier, A. DuChesne, G.H. Willital, P. Bruckner (2001) Endochondral ossification of costal cartilage is arrested after chondrocytes have reached hypertrophic stage of late differentiation. Matrix Biol 19: 707-715

Ballock, R.T., A. Heydemann, L.M. Wakefield, K.C. Flanders, A.B. Roberts, M.B. Sporn (1993) TGF- $\beta 1$ prevents hypertrophy of epiphyseal chondrocytes: regulation of gene expression for cartilage matrix proteins and metalloproteases. Dev Biol 158: 414-429. 
Ballock, R.T., R.J. O’Keefe (2003) Physiology and pathophysiology of the growth plate. Birth Defects Res C Embryo Today 69: 123-143.

-Barry, F., R.E. Boynton, B. Liu, J.M. Murphy (2001) Chondrogenic differentiation of mesenchymal stem cells from bone marrow: differentiation-dependent gene expression of matrix components. Exp Cell Res 268: 189200.

Cheung, J.O., M.C. Hillarby, S. Ayad, J.A. Hoyland, C.J. Jones, J. Denton, J.T. Thomas, G.A. Wallis, M.E. Grant (2001) A novel cell culture model of chondrocyte differentiation during mammalian endochondral ossification. J Bone Miner Res 16: 309-318.

Farquharson, C., C.C. Whitehead (1995) Differentiation and mineralization in chick chondrocytes maintained in a high cell density culture: a model for endochondral ossification. In Vitro Cell Dev Biol Anim 31: 288294.

Goldring, M.B., K. Tsuchimochi, K. Ijiri (2006) The control of chondrogenesis. J Cell Biochem 97: 33-44.

-Hunziker, E.B., E. Kapfinger, C. Saager (1999) Hypertrophy of growth plate chondrocytes in vivo is accompanied by modulations in the activity state and surface area of their cytoplasmic organelles. Histochem Cell Biol 112: 115-123.

- Ichinose, S., M. Tagami, T. Muneta, I. Sekiya (2005) Morphological examination during in vitro cartilage formation by human mesenchymal stem cells. Cell Tissue Res 322: 217-226.

-Iwamoto, M., A. Shimazu, M. Pacifici (1995) Regulation of chondrocyte maturation by fibroblast growth factor-2 and parathyroid hormone. J Orthop Res 13: 838-845.

Johnstone, B., T.M. Hering, A.I. Caplan, V.M. Goldberg, J.U. Yoo (1998) In vitro chondrogenesis of bone marrow-derived mesenchymal progenitor cells. Exp Cell Res 238: 265272.

-Johnstone, B., J.U. Yoo (1999) Autologous mesenchymal progenitor cells in articular cartilage repair. Clin Orthop Relat Res 367 (suppl): S156-S162.
Leboy, P.S., T.A. Sullivan, M. Nooreyazdan, R.A. Venezian (1997) Rapid chondrocyte maturation by serum-free culture with BMP-2 and ascorbic acid. J Cell Biochem 66: 394-403.

Li, W.J., R. Tuli, C. Okafor, A. Derfoul, K.G. Danielson, D.J. Hall, R.S. Tuan (2005) A threedimensional nanofibrous scaffold for cartilage tissue engineering using human mesenchymal stem cells. Biomaterials 26: 599-609.

Lisignoli, G., S. Cristino, A. Piacentini, S. Toneguzzi, F. Grassi, C. Cavallo, N. Zini, L. Solimando, N. Mario Maraldi, A. Facchini (2005) Cellular and molecular events during chondrogenesis of human mesenchymal stromal cells grown in a three-dimensional hyaluronan based scaffold. Biomaterials 26 5677-5686.

Mackay, A.M., S.C. Beck, J.M. Murphy, F.P. Barry, C.O. Chichester, M.F. Pittenger (1998) Chondrogenic differentiation of cultured human mesenchymal stem cells from marrow. Tissue Eng 4: 415-428.

Mello, M.A., R.S. Tuan (2006) Effects of TGF- $\beta 1$ and triiodothyronine on cartilage maturation: in vitro analysis using long-term highdensity micromass cultures of chick embryonic limb mesenchymal cells. J Orthop Res 24: 2095-2105.

Mueller, M.B., R.S. Tuan (2008) Functional characterization of hypertrophy in chondrogenesis of human mesenchymal stem cells. Arthritis Rheum 58: 1377-1388.

Mwale, F., P.L. Girard-Lauriault, H.T. Wang, S. Lerouge, J. Antoniou, M.R. Wertheimer (2006a) Suppression of genes related to hypertrophy and osteogenesis in committed human mesenchymal stem cells cultured on novel nitrogen-rich plasma polymer coatings. Tissue Eng 12: 2639-2647.

Mwale, F., D. Stachura, P. Roughley, J. Antoniou (2006b) Limitations of using aggrecan and type $\mathrm{X}$ collagen as markers of chondrogenesis in mesenchymal stem cell differentiation. J Orthop Res 24: 1791-1798.

Noth, U., R. Tuli, A.M. Osyczka, K.G. Danielson, R.S. Tuan (2002) In vitro engineered cartilage constructs produced by press-coating biodegradable polymer with human mesenchymal stem cells. Tissue Eng 8: 131-144.
Okubo, Y., A.H. Reddi (2003) Thyroxine downregulates Sox9 and promotes chondrocyte hypertrophy. Biochem Biophys Res Commun 306: 186-190.

Pelttari, K., A. Winter, E. Steck, K. Goetzke, T. Hennig, B.G. Ochs, T. Aigner, W. Richter (2006) Premature induction of hypertrophy during in vitro chondrogenesis of human mesenchymal stem cells correlates with calcification and vascular invasion after ectopic transplantation in SCID mice. Arthritis Rheum 54: 3254-3266.

Poole, A.R., Y. Matsui, A. Hinek, E.R. Lee (1989) Cartilage macromolecules and the calcification of cartilage matrix. Anat Rec 224: 167179.

Quarto, R., G. Campanile, R. Cancedda, B. Dozin (1997) Modulation of commitment, proliferation, and differentiation of chondrogenic cells in defined culture medium. Endocrinology 138: 4966-4976.

-Sekiya, I., J.T. Vuoristo, B.L. Larson, D.J. Prockop (2002) In vitro cartilage formation by human adult stem cells from bone marrow stroma defines the sequence of cellular and molecular events during chondrogenesis. Proc Natl Acad Sci USA 99: 4397-4402.

-Shimizu, H., S. Yokoyama, H. Asahara (2007) Growth and differentiation of the developing limb bud from the perspective of chondrogenesis. Dev Growth Differ 49: 449-454.

Song, L., D. Baksh, R.S. Tuan (2004) Mesenchymal stem cell-based cartilage tissue engineering: cells, scaffold and biology. Cytotherapy 6: 596-601.

Tuli, R., S. Tuli, S. Nandi, X. Huang, P.A. Manner, W.J. Hozack, K.G. Danielson, D.J. Hall, R.S. Tuan (2003) Transforming growth factor-beta-mediated chondrogenesis of human mesenchymal progenitor cells involves $\mathrm{N}$ cadherin and mitogen-activated protein $\mathrm{ki}$ nase and Wnt signaling cross-talk. J Biol Chem 278: 41227-41236.

Yoo, J.U., T.S. Barthel, K. Nishimura, L. Solchaga, A.I. Caplan, V.M. Goldberg, B. Johnstone (1998) The chondrogenic potential of human bone-marrow-derived mesenchymal progenitor cells. J Bone Joint Surg Am 80: 17451757. 